\title{
Correction of Coronal Imbalance in Degenerative Lumbar Spine Disease Following Direct Lateral Interbody Fusion (DLIF)
}

\author{
Ju Seong Kim, Hyo Sang Lee, Dong Ah Shin, Keung Nyun Kim, Do Heum Yoon \\ Department of Neurosurgery, Yonsei University College of Medicine, Severance Hospital, \\ The Spine and Spinal Cord Institute, Seoul, Korea
}

\begin{abstract}
Objective: The authors have recently been using a surgical technique of minimally invasive direct lateral interbody fusion (DLIF) for correcting of coronal imbalance. The purpose of this study was to evaluate the surgical outcome and complication of DLIF.

Methods: We undertook retrospective analysis of a consecutive series of 8 DLIF procedures in Degenerative lumbar spine disease since May 2011. Four patients underwent DLIF only, and the others underwent combined DLIF and posterior fixation. Data on intra- and postoperative complications were collected. The pre- and postoperative X-rays were reviewed. We investigated coronal deformity, Cobb's angle, and apical vertebral translation (AVT). The mean follow-up period was months with a range of 2 to 8 months.

Results: A mean preoperative coronal Cobb's angle was $21.8^{\circ}$ (range $11.5-32.4^{\circ}$ ). Following after DLIF, the mean Cobb's angle was decreased to $13.0^{\circ}$ (range 2.9-21.5 ). Following additional posterior screw fixation, mean Cobb's angle was further decreased to $7.4^{\circ}$ (range 2.9-13.2 ). A mean preoperative AVT was $2.0 \mathrm{~cm}$ (range 0.6-3.5 cm), and improved to $1.4 \mathrm{~cm}$ (range $0.3-2.4 \mathrm{~cm}$ ) and $0.8 \mathrm{~cm}$ (range $0.2-1.8 \mathrm{~cm}$ ) postoperatively (DLIF and, posterior fixation respectively). One patient $(12.5 \%)$ showed cage migration during follow-up period. Two patients (25\%) developed motor weakness, and 4 patients (50\%) experienced postoperative thigh paresthesias or dysesthesias. During follow up period, motor weakness had resolved in 1 patient. Sensory symptoms were improved in all patients at the last follow-up.

Conclusion: Degenerative lumbar disease can be effectively corrected by DLIF with acceptable complications.
\end{abstract}

Key Words: Degenerative lumbar disease $\cdot$ Coronal imbalance $\cdot$ DLIF $\cdot$ Lumbosacral plexus $\cdot$ Postoperative complication

\section{INTRODUCTION}

Recently surgical trends are toward minimally invasive operations, which decreases blood loss and post-operative pain and enables patients to return to their ordinary life after short hospital stays ${ }^{4,714,20,28)}$. The first laparoscopic lumbar discectomy was introduced in 1991 by Obenchain ${ }^{17}$. Following that, new surgical methods such as transforaminal lumbar interbody fusion (TLIF) and direct lateral interbody fusion (DLIF) were introduced for degenerative lumbar spine disease.

DLIF, also called as Extreme Lateral Interbody Fusion $(\mathrm{XLIF})^{19}$, is a method of maximizing the advantages of ante-

- Received: April 13, 2012 - Revised: September 4, 2012

- Accepted: September 26, 2012

Corresponding Author: Keung Nyun Kim, MD

Department of Neurosurgery, Yonsei University College of Medicine,

134 Shinchon-Dong, Seodaemoon-Gu, Seoul 120-752, Korea

Tel: +82-2-2228-2152, Fax: +82-2-393-9979

Email: knkim@yuhs.ac rior lumbar interbody fusion (ALIF) and improving accessibility to anterior spinal body that facilitates minimum invasive treatment by transpsoas approach. This technique has been used to treat degenerative lumbar disease including degenerative scoliosis ${ }^{1,4,6,13)}$. The authors have recently been using a surgical technique of minimally invasive direct lateral interbody fusion (DLIF) for correcting coronal imbalance. The purpose of this study was to evaluate surgical outcome and complications of DLIF for Degenerative lumbar spine disease.

\section{MATERIALS AND METHODS}

\section{Patient population}

This study included a consecutive series of 8 patients (mean age 65.8, range 51-76, $\mathrm{M}: \mathrm{F}=2: 7$ ) who underwent $\mathrm{DLIF}$ in our institute between May, 2011 and Feb, 2012. Among a total of 31 DLIF cases, 8 patients underwent DLIF for Degenerative lumbar spine disease. Four patients underwent DLIF only, and the others underwent combined DLIF and posterior 
fixation(Table 1). Mean follow up period was 3 months

\section{Surgical procedures}

The patient is placed on a surgical table in a true $90^{\circ}$ lateral decubitus position and taped in this position. C-arm guided cross table anterior-posterior (AP) image help to confirm the true $90^{\circ}$ position. Surgical table and patient are flexed in such a way as to increase the distance between the iliac crest and the rib cage, especially at L4-L5. It is helpful to place a pillow or roll under the contralateral flank ${ }^{19)}$. EMG electrodes are placed for intra-operative monitoring as previous reports. Intra-operative

Table 1. Summary of demographic profiles

\begin{tabular}{lc}
\hline \hline Variables & \multicolumn{1}{c}{ Data } \\
\hline Demographics & \\
Age (years) & $65.8 \pm 8.5(51-76)$ \\
Gender (M:F) & $2: 7$ \\
Diagnosis & \\
Degenerative scoliosis & $6(75 \%)$ \\
Segmental scoliosis & $2(25 \%)$ \\
Posterior instrumentation & \\
DLIF only & $4(50 \%)$ \\
DLIF+PSF & $4(50 \%)$ \\
\hline
\end{tabular}

DLIF; Direct Lateral Interbody Fusion

PSF; Pedicle Screw Fixation
EMG monitoring system (NIM-Spine, Medtronic Sofamor Danek, TN, USA) is installed to estimate close proximity of the lumbosacral plexus to the advancing tubular dilator ${ }^{3,5,9,27)}$. A 4-cm-sized oblique incision is made and retroperitoneal finger dissection is performed. Through this small incision, atraumatic tissue dilators and an expandable retractor are inserted, which can be the working portal ${ }^{19)}$. We check for normal EMG and then conduct discectomy. During discectomy, we check that it is released to the opposite annulus. After sufficient discectomy, interbody fusion is conducted using DLIF cage. In most cases, we make 1 incision for multilevel DLIF but for some patients another incision is made.

We use Clydesdale cages (Medtronic Sofamor Danek, TN, USA) for DLIF, with heights from 8 to $12 \mathrm{~mm}$, lengths from 45 to $55 \mathrm{~mm}$ and a total lordosis angle of $0^{\circ}, 6^{\circ}$. All DLIF grafts were packed with $\beta$-tricalcium phosphate $\left(\mathrm{ChronOS}^{\square}\right.$ strip, Synthes, PA, USA) and demineralized bone matrix (DBX ${ }^{\square}$ putty, Synthes, PA, USA). Since DLIF cannot be conducted to $\mathrm{L} 5 / \mathrm{S} 1$ which is covered by sacral crest, for the group who were receiving pedicle screw fixation, PLIF was followed by pedicle screw fixation to this lesion.

\section{Radiologic evaluation}

L-spine series X-ray was examined to compare the 8 patients' pre and post operative status and coronal Cobb's angle and apical vertebral translation (AVT) were compared to check the

Table 2. Summary of surgical profiles (Cobb's angle/AVT)

\begin{tabular}{llccccc}
\hline \hline No. & Age/Sex & DLIF & PSF & Pre op $(\% / \mathrm{cm})$ & DLIF $(\% / \mathrm{cm})$ & PSF $(\% / \mathrm{cm})$ \\
\hline 1 & $69 / F$ & L1-L4 & & $28.2 \pm 3.2(25.3-31.7) /$ & $20.7 \pm 1.8(18.8-22.3) /$ & \\
& & & & $3.0 \pm 0.17(2.8-3.1)$ & $2.3 \pm 0.20(2.1-2.5)$ & \\
$2^{*}$ & $64 / F$ & L2-L4 & L1-L5 & $25.7 \pm 1.9(24.0-27.8) /$ & $21.5 \pm 1.9(19.8-23.5) /$ & $9.2 \pm .0 .3(8.9-9.5) /$ \\
& & & & $2.6 \pm 0.15(2.4-2.7)$ & $2.4 \pm 0.11(2.4-2.6)$ & $0.6 \pm 0.05(0.6-0.7)$ \\
$3^{*}$ & $67 / F$ & L1-L4 & T12-L5 & $26.8 \pm 1.5(25.4-28.3) /$ & $23.0 \pm 2.0(20.8-24.5) /$ & $13.2 \pm 1.1(12.3-14.4) /$ \\
& & & & $2.9 \pm 0.35(2.5-3.2)$ & $2.0 \pm 0.15(1.9-2.2)$ & $1.8 \pm 0.23(1.5-1.9)$ \\
$4^{*}$ & 74/F & L1-L4 & T10-S1 & $11.5 \pm 1.8(10.2-13.5) /$ & $8.3 \pm 0.8(7.9-9.3) /$ & $2.9 \pm 0.4(2.5-3.2) /$ \\
& & & & $0.7 \pm 0.09(0.6-0.9)$ & $0.4 \pm 0.05(0.4-0.5)$ & $0.2 \pm 0.05(0.1-0.2)$ \\
$5^{*}$ & $51 / M$ & L2-L5 & T12-L5 & $32.4 \pm 2.4(30.3-35.0) /$ & $11.7 \pm 0.5(11.3-12.2) /$ & $4.4 \pm 0.5(3.8-4.8) /$ \\
& & & & $3.5 \pm 0.20(3.3-3.7)$ & $2.0 \pm 0.15(1.8-2.1)$ & $0.6 \pm 0.5(0.6-0.7)$ \\
6 & $56 / F$ & L3-L5 & & $17.2 \pm 0.8(16.5-18.1) /$ & $10.6 \pm 0.8(9.7-11.5) /$ & \\
& & & & $0.6 \pm 0.06(0.6-0.7)$ & $0.3 \pm 0.06(0.3-0.4)$ & \\
7 & 76/M & L2-L4 & & $20.9 \pm 0.6(20.2-21.4) /$ & $15.1 \pm 2.5(12.3-17.2) /$ & \\
& & & & $1.4 \pm 0.15(1.3-1.6)$ & $1.0 \pm 0.15(0.9-1.2)$ & \\
8 & $69 / F$ & L2-L4 & & $13.9 \pm 1.1(12.6-14.8) /$ & $6.6 \pm 0.4(6.2-7.0) /$ & \\
& & & & $1.7 \pm 0.06(1.6-1.7)$ & $1.0 \pm 0.25(0.7-1.2)$ & \\
\hline
\end{tabular}

*DLIF+PSF

DLIF; Direct Lateral Interbody Fusion, PSF; Pedicle Screw Fixation, AVT; Apical Vertebral Translation 
improvement of degenerative lumbar scoliosis. Cobb's angle is an index to determine the severity of sagittal or coronal deformity by measuring the angle made by 2 perpendicular lines from the extended lines of upper and lower endplate with the severest scoliosis $^{26}$.

AVT is an indicator of determining the degree of scoliosis by measuring the distant from the center sacral vertebral line (CSVL) to the midpoint of the apical vertebral body of the curve $^{26)}$.

\section{Data analysis}

Measurements were collected and analyzed by using software (Microsoft Excel, Microsoft Corp., WA, USA). A p $<0.05$ was regarded as statistical significance.

\section{RESULTS}

\section{Demographics}

In our experience, 31 cases of DLIF were conducted since May, 2011 and 8 cases among them were done to the patients with coronal imbalance. 4 patients underwent DLIF procedures and the other 4 underwent additional pedicle screw fixation (PSF) by posterior approach after transpsoas DLIF.

\section{Radiologic outcomes}

The pre-operative average Cobb's angle of all patients is $21.8^{\circ}$ (range $11.5-32.4^{\circ}$ ) and the average of 4 patients who operated additional PSF is $24.1^{\circ}$, which shows a little more serious scoliosis than that of the entire. After surgical manage-

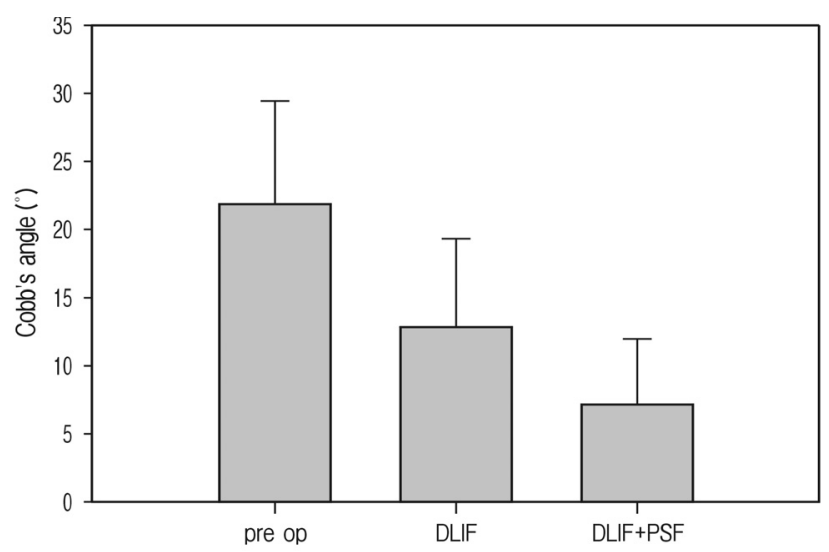

Fig. 1. Correction of Cobb's angle; pre op mean Cobb's angle was $21.8^{\circ}$ that improved to $13^{\circ}$ after DLIF, and more improved to $7.3^{\circ}$ after additional pedicle screw fixation. ment for scoliosis, all 8 patients who were received DLIF got better and their average Cobb's angle improved to $13^{\circ}$ (range 2.9-21.5 ). The 4 patients who were received additional PSF after DLIF, saw their average final Cobb's angle much improved to $7.4^{\circ}$ (range $2.9-13.2^{\circ}$ )(Fig. 1).

As with Cobb's angle, AVT also showed marked improvement after operation. Total mean AVT was $2.0 \mathrm{~cm}$ (range $0.6-3.5 \mathrm{~cm}$ ) before surgery, but in which improved to $1.4 \mathrm{~cm}$ (range 0.3-2.4 $\mathrm{cm})$ after DLIF only and the additional PSF group was much improved to $0.8 \mathrm{~cm}($ range $0.2-1.8 \mathrm{~cm}$ ) (Fig. 2).

Of course, it's expected that the additional PSF group has much improvement of Cobb's angle and AVT, but the standalone DLIF group shows sufficient correction of coronal imbalance. And standalone DLIF group's correction rate of Cobb's angle was stiffer than the additional PSF group's rate.

\section{Complications}

Some patients had cage-related complications. Two cases of cage subsidence were detected in standalone DLIF group, and one case of cage migration was detected. Cage migration patients who received additional expansive posterior pedicle screw fixation for prevent spinal instability (Fig. 3). This patient was included additional PSF group in this study.

Also, lumbosacral plexus related complications were detected. Two patients (25\%) developed motor weakness, and 4 patients (50\%) experienced postoperative thigh paresthesias or dysesthesias. But, motor weakness had recovered in few weeks for 1 patient. Sensory symptoms were improved in all patients at the last follow-up.

No cases had serious complications and no severe morbidity and mortality resulted from Degenerative lumbar spine disease.

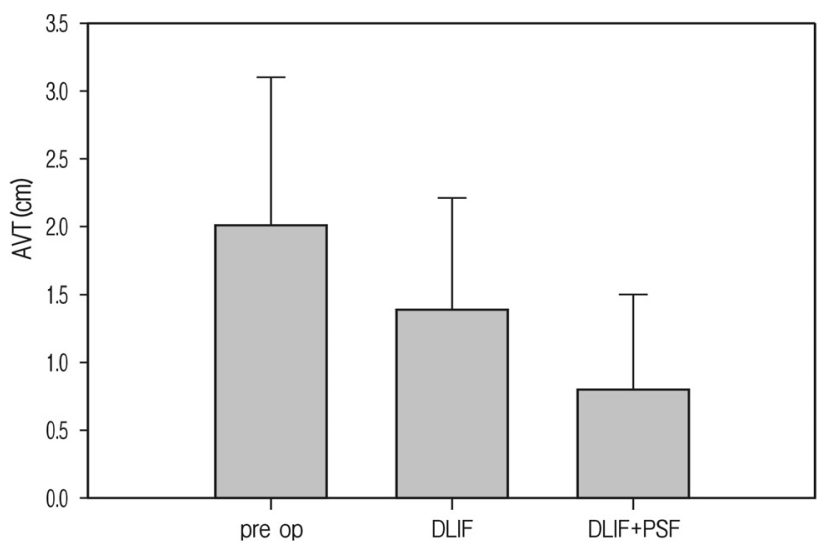

Fig. 2. Correction of Apical Vertebral Translation (AVT); pre op mean $\mathrm{AVT}$ was $2.0 \mathrm{~cm}$ that improved to $1.4 \mathrm{~cm}$ after DLIF, and more improved to $0.8 \mathrm{~cm}$ after additional pedicle screw fixation. 

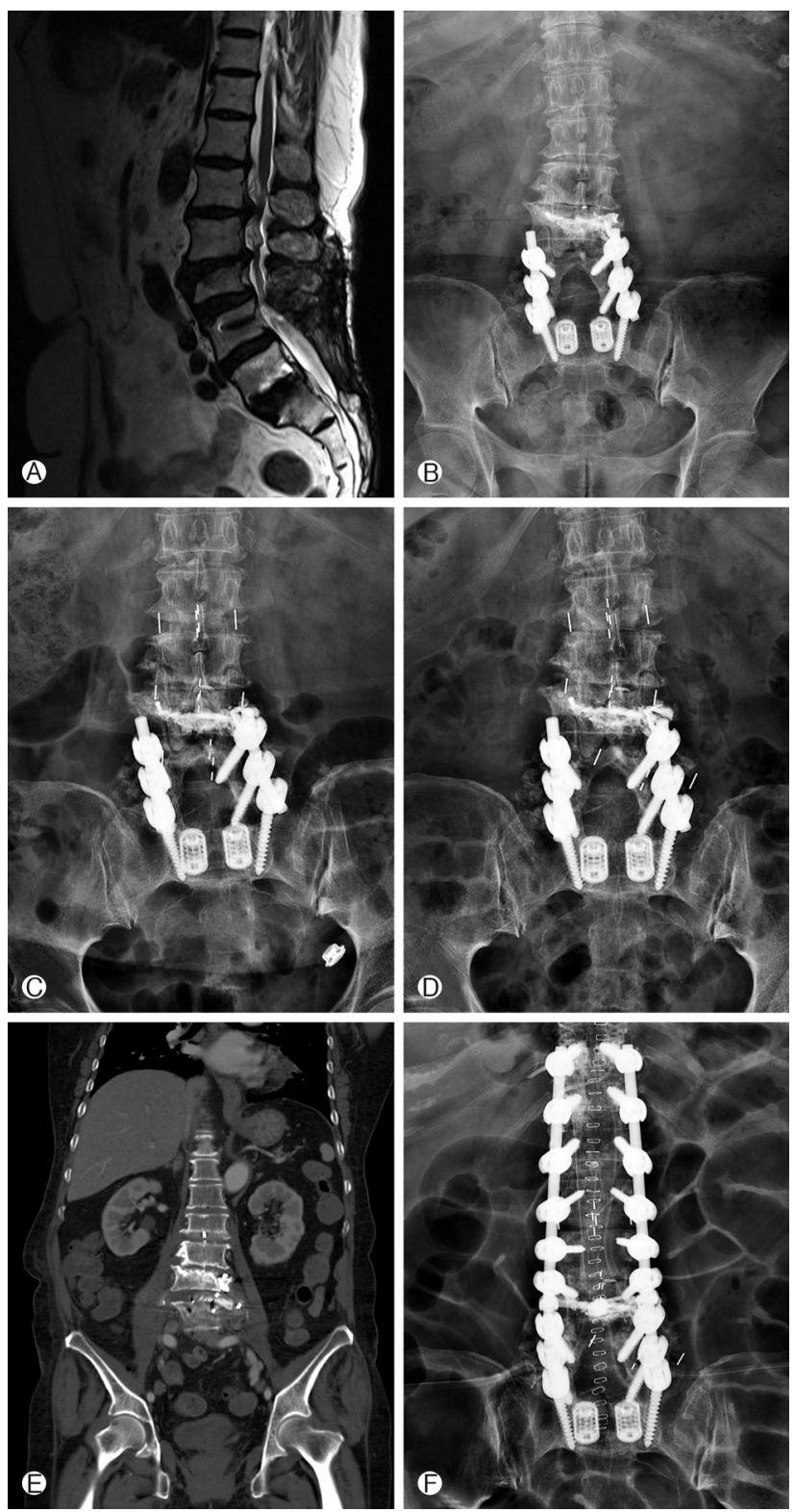

Fig. 3. Case 4 patient is 74-year-old female; she has been operated PLIF L5-S1 and pedicle screw fixation on L4-5-S1 in 2009. And L3 percutaneous vertebroplasty was done. (A, B) She visited our hospital due to topping off and segmental scoliosis L1-2-3-4. (C) Performed DLIF surgery. (D) After 1 month follow up L-spine X-ray and (E) abdominal CT coronal image was shown L3-4 DLIF cage migration. (F) Finally, We did expansive pedicle screw fixation for prevent spinal instability (PLIF; Posterior Lumbar Interbody Fusion, DLIF; Direct Lateral Interbody Fusion).

\section{DISCUSSION}

The lateral, transpsoas approach was first introduced in 2001 by Pimenta ${ }^{21}$. It was developed into XLIF in 2006 by
Ozgur ${ }^{19)}$ and presented in Korea in May 2011 under the name of DLIF. DLIF approaches to vertebral body directly and can be conducted without any help from an extra-surgeon who performs an anterior abdominal approach like ALIF ${ }^{1,10,19,24)}$. It also has the advantage of enabling a surgical approach without any risk to great vessels ${ }^{2)}$ or retrograde ejaculation ${ }^{22)}$ which are potential problems in anterior approach ${ }^{1,10,19)}$. The fusion surface area is enhanced by using a much larger and higher cage than in historical PLIF or TLIF, thus improving the reduction rate in degenerative spine disease ${ }^{8,18,23)}$. In addition, it is effective in reducing peri-operative bleeding and operation time with minimal invasive technique ${ }^{19)}$. It has also, less postoperative pain, leading to early recovery and faster return to ordinary life ${ }^{16}$. DLIF technique is very effective and has many advantages.

Minimally invasive scoliosis correction is a very challenging to a spinal deformity surgeon. But traditional open extensive scoliosis correction was hard work and very dangerous surgery. For the patient's safety and appropriated surgical correction of deformity, minimally invasive deformity correction technique was needed. DLIF provide for bilateral annulus release and after disectomy, placement of a large interbody spacer that allows correction of coronal deformity. This reason can make the people believe DLIF is valuable tool for scoliosis correction. Anand et al report correction of Cobb's angle form $18.93^{\circ}$ to $6.19^{\circ}$ through minimal invasive multilevel percutaneous screw fixation ${ }^{1)}$. Some studies compare combined transpsoas approach and traditional posterior approach for scoliosis treatment. Tormenti et al report radiographic outcome as the Cobb's angle and AVT were significantly improved in patients who underwent a combined transpsoas and posterior approach $^{26}$. In this study, we retrospectively reviewed radiograph to contrast standalone DLIF and DLIF with open posterior PSF for scoliosis correction. According to our data, standalone DLIF improved Cobb's angle by $40.3 \%$ (from 21.8 to 13.0) and AVT by $30 \%$ (from $2.0 \mathrm{~cm}$ to $1.4 \mathrm{~cm}$ ).

Despite of this effective correction, standalone DLIF has some complication, such as, subsidence of cage and, cage migration. Marchi et al reported standalone DLIF subsidence rate was $17.3 \%{ }^{15)}$. Recently, minimally invasive percutaneous pedicle screw fixation systems were developed well. Long-level percutenous screw fixation is available now. So the combination of DLIF and percutaneous screw fixation is a good tool for degenerative scoliosis correction. This combination can prevent cage related complication such as cage subsidence and migration.

Another major DLIF complication is lumbosacral plexus injury. In our experience, transient thigh sensory change (25\%) and psoas muscle weakness (12.5\%) rate were similar to previous literature reports ${ }^{11,12,25)}$. The key to solving this problem 
is less damage to the psoas muscle during finger transpsoas disse ction and retracted dilator insertion. And close observation of intra-operative EMG monitoring was needed.

As for our result, standalone DLIF gets enough reduction of spinal alignment and is a good treatment tool for segmental scoliosis $^{15)}$. To prevent cage related complications, a combination of DLIF and additional reinforcement surgery is the best choice for minimal invasive scoliosis correction.

Our study is limited by small numbers and lack of sufficient experiences. We need longer follow up data.

\section{CONCLUSION}

Degenerative lumbar spine disease with coronal imbalance can be effectively corrected by DLIF with acceptable complication rates.

\section{REFERENCES}

1. Anand N, Baron EM, Thaiyananthan G, Khalsa K, Goldstein TB: Minimally invasive multilevel percutaneous correction and fusion for adult lumbar degenerative scoliosis: a technique and feasibility study. J Spinal Disord Tech 21(7):459-467, 2008

2. Baker JK, Reardon PR, Reardon MJ, Heggeness MH: Vascular injury in anterior lumbar surgery. Spine (Phila Pa 1976) 18(15): 2227-2230, 1993

3. Banagan K, Gelb D, Poelstra K, Ludwig S: Anatomic mapping of lumbar nerve roots during a direct lateral transpsoas approach to the spine: acadaveric study. Spine (Phila Pa 1976) 36(11): E687-691, 2011

4. Benglis DM, Elhammady MS, Levi AD, Vanni S: Minimally invasive anterolateral approaches for the treatment of back pain and adult degenerative deformity. Neurosurgery 63(3 Suppl):191-196, 2008

5. Benglis DM, Vanni S, Levi AD: An anatomical study of the lumbosacral plexus as related to the minimally invasive transpsoas approach to the lumbar spine. J Neurosurg Spine 10(2):139-144, 2009

6. Dakwar E, Cardona RF, Smith DA, Uribe JS: Early outcomes and safety of the minimally invasive, lateral retroperitoneal transpsoas approach for adult degenerative scoliosis. Neurosurg Focus 28(3):E8, 2010

7. Eck JC, Hodges S, Humphreys SC: Minimally invasive lumbar spinal fusion. J Am Acad Orthop Surg 15(6):321-329, 2007

8. Elowitz EH, Yanni DS, Chwajol M, Starke RM, Perin NI: Evaluation of indirect decompression of the lumbar spinal canal following minimally invasive lateral transpsoas interbody fusion: radiographic and outcome analysis. Minim Invasive Neurosurg 54(5-6): 201-206, 2011

9. Galloway GM: Direct lateral transpsoas approach to interbody fusion-may be risky after all. J Cin Neurophysiol 28(6):605-606, 2011

10. Gumbs AA, Shah RV, Yue JJ, Sumpio B: The open anterior paramedian retroperitoneal approach for spine procedures. Arch Surg 140(4):339-343, 2005

11. Houten JK, Alexandre LC, Nasser R, Wollowick AL: Nerve injury during the transpsoas approach for lumbar fusion. J Neurosurg Spine 15(3):280-284, 2011

12. Karikari IO, Nimjee SM, Hardin CA, Hughes BD, Hodges TR, Mehta AI, et al:Extreme lateral interbody fusion approach for isolated thoracic and thoracolumbar spine diseases: Initial clinical experience and early outcomes. J Spinal Disord Tech 24(6): 368-375, 2011

13. Knight RQ, Schwaegler P, Hanscom D, Roh J: Direct lateral lumbar interbody fusion for degenerative conditions: early complication profile. J Spinal Disord Tech 22(1):34-37, 2009

14. Lee YG CJ, Park JS: Clinical outcome of minimally invasive tubular retractor assisted microscopic discectomy in far lateral lumbar disc herniation. Korean J Spine 7(3):155-160, 2010

15. Marchi L, Abdala N, Oliveira L, Amaral R, Coutinho E, Pimenta L: Stand-alone lateral interbody fusion for the treatment of lowgrade degenerative spondylolisthesis. Scientific World Journal 2012:456346, 2012

16. Mobbs RJ, Sivabalan P, Li J: Minimally invasive surgery compared to open spinal fusion for the treatment of degenerative lumbar spine pathologies. J Clin Neurosci 19(6):829-835, 2012

17. Obenchain TG: Laparoscopic lumbar discectomy: case report. J Laparoendosc Surg 1(3):145-149, 1991

18. Oliveira L, Marchi L, Coutinho E, Pimenta L: A radiographic assessment of the ability of the extreme lateral interbody fusion procedure to indirectly decompress the neural elements. Spine (Phila Pa 1976) 35 (26 Suppl):S331-337, 2010

19. Ozgur BM, Aryan HE, Pimenta L, Taylor WR: Extreme lateral interbody fusion (xlif): anovel surgical technique for anterior lumbar interbody fusion. Spine J 6(4):435-443, 2006

20. Park Y, Ha JW: Comparison of one-level posterior lumbar interbody fusion performed with a minimally invasive approach or a traditional open approach. Spine (Phila Pa 1976) 32(5):537543, 2007

21. Pimenta L: Lateral endoscopic transpsoas retroperitoneal approach for lumbar spine surgery: VIII Brazilian Spine Society Meeting. Belo Horizonte, Minas Gerais, Brazil, 2001

22. Rajaraman V, Vingan R, Roth P, Heary RF, Conklin L, Jacobs GB: Visceral and vascular complications resulting from anterior lumbar interbody fusion. J Neurosurg 91(1 Suppl):60-64, 1999

23. Richards JC, Majumdar S, Lindsey DP, Beaupre GS, Yerby SA: The treatment mechanism of an interspinous process implant for lumbar neurogenic intermittent claudication. Spine (Phila Pa 1976) 30(7):744-749, 2005

24. Riedel CJ: Open anterior lumbar interbody fusion. Clin Neurosurg 47:534-540, 2000

25. Rodgers WB, Cox CS, Gerber EJ: Early complications of extreme lateral interbody fusion in the obese. J Spinal Disord Tech 23 (6):393-397, 2010

26. Tormenti MJ, Maserati MB, Bonfield CM, Okonkwo DO, Kanter AS:Complications and radiographic correction in adult scoliosis following combined transpsoas extreme lateral interbody fusion and posterior pedicle screw instrumentation. Neurosurg Focus 28(3):E7, 2010

27. Uribe JS, Vale FL, Dakwar E: Electromyographic monitoring and its anatomical implications in minimally invasive spine surgery. Spine (Phila Pa 1976) 35(26 Suppl):S368-374, 2010

28. Wang MY, Anderson DG, Poelstra KA, Ludwig SC: Minimally invasive posterior fixation. Neurosurgery 63(3 Suppl):197-203, 2008 